\title{
Determinantes sociais da saúde de gestantes acompanhadas no pré- natal de alto risco
}

\author{
Social determinants of health of high-risk pregnant women during prenatal follow-up
}

\section{Como citar este artigo:}

Gadelha IP, Diniz FF, Aquino PS, Silva DM, Balsells MMD, Pinheiro AKB. Social determinants of health of high-risk pregnant women during prenatal follow-up. Rev Rene. 2020;21:e42198. DOI: https://doi.org/10.15253/2175-6783.20202142198

(DIvyna Pires Gadelha ${ }^{1}$
(D) Flaviane Fabricio Diniz ${ }^{1}$
(D) Priscila de Souza Aquino ${ }^{1}$
(D) Denise Montenegro da Silva
(D) Marianne Maia Dutra Balsells ${ }^{1}$
(D) Ana Karina Bezerra Pinheiro ${ }^{1}$

${ }^{1}$ Universidade Federal do Ceará.

Fortaleza, CE, Brasil.

\author{
Autor correspondente: \\ Ivyna Pires Gadelha \\ Rua Alexandre Baraúna, 1115 - Rodolfo Teófilo. \\ CEP: 60430-160. Fortaleza, CE, Brasil. \\ E-mail: ivynapires@gmail.com
}

\begin{abstract}
RESUMO
Objetivo: avaliar os determinantes sociais da saúde de gestantes acompanhadas no pré-natal de alto risco. Métodos: estudo observacional e transversal, realizado com 276 gestantes de alto risco. A coleta deu-se mediante aplicação de questionário estruturado acerca dos determinantes sociais da saúde, além de dados clínicos e obstétricos das mulheres. Os dados foram analisados através do programa estatístico Jamovi®, versão 0.9 , e discutidos segundo o Modelo de Dahlgren e Whitehead. Resultados: as condições individuais; o comportamento e estilo de vida; a rede social e comunitária; e a condição de vida, tais como: trabalho, saúde, educação, saneamento básico e habitação podem constituir fator de risco ou de proteção à saúde da gestante. Conclusão: determinantes como idade, raça, tipo de gestação, nível educacional, a situação de moradia e o acesso aos serviços de saúde foram considerados favoráveis à saúde da maioria das gestantes, porém, a prevalência de gravidez não planejada como comportamento desfavorável. Descritores: Gravidez de Alto Risco; Assistência Pré-Natal; Determinantes Sociais da Saúde.
\end{abstract}

\section{ABSTRACT}

Objective: to evaluate the social determinants of health of high-risk pregnant women during prenatal follow-up. Methods: observational and cross-sectional study with 276 high-risk pregnant women. Data were collected by applying a structured questionnaire about the social determinants of health, as well as clinical and obstetric data from highrisk pregnant women. Data were analyzed using the Jamovi ${ }^{\circ}$ statistical software, version 0.9 , and discussed according to the Dahlgren and Whitehead's Model. Results: individual conditions; behavior and lifestyle; social and community network; and the condition of life, such as work, health, education, sanitation and housing may constitute a risk or health protection factor for pregnant women. Conclusion: determinants such as age, race, type of pregnancy, educational level, housing situation and access to health services were considered favorable to the health of most pregnant women, but the prevalence of unplanned pregnancy as unfavorable behavior.

Descriptors: Pregnancy, High-Risk; Prenatal Care; Social Determinants of Health. 


\section{Introdução}

A gestação é um fenômeno fisiológico na vida da mulher, no entanto, algumas condições podem representar maior probabilidade de resultados desfavoráveis para a mãe e/ou para o feto/recém-nascido, insinuando aumento do risco gestacional.

$\mathrm{O}$ alto risco gestacional pode referir-se aos fatores individuais, às condições socioeconômicas desfavoráveis, às doenças maternas prévias, à história reprodutiva anterior e/ou à gestação atual ${ }^{(1)}$. 0 alto risco gestacional compõe cerca de $15,0 \%$ das gestações ${ }^{(2)}$.

A assistência pré-natal de qualidade favorece o reconhecimento dos fatores desfavoráveis ao seguimento da gestação - tanto aqueles relacionados às condições clínicas como associados às condições básicas de vida - e permite a avaliação do risco gestacional de forma contínua, além da intervenção precoce, o que favorece melhores resultados de saúde ${ }^{(3)}$. Nesse contexto, considera-se essencial conhecer as condições que podem estar diretamente relacionadas à situação de saúde e doença.

Sob essa ótica, evidencia-se a relevância da avaliação dos determinantes sociais da saúde, construto relacionado aos comportamentos individuais, às condições de vida e de trabalho e à macroestrutura econômica, social e cultural. 0 modelo de avaliação dos determinantes sociais da saúde proposto por Dahlgren e Whitehead descreve cinco camadas e ratifica a importância dos aspectos não clínicos sobre o estado de saúde dos indivíduos e das populações ${ }^{(4)}$.

Devido à lacuna na literatura acerca dos determinantes sociais da saúde de gestantes de alto risco, a presente investigação faz-se essencial, uma vez que possibilita aos profissionais identificar as condições de vulnerabilidade em que as gestantes estão inseridas e, assim, conduzir suas ações para a promoção da saúde e prevenção de agravos nessa população. Ademais, os achados desse estudo poderão conduzir gestores de saúde no direcionamento de ações que fortaleçam a integralidade do cuidado às gestantes. Nesse contexto, tem-se como pergunta norteadora: quais os principais determinantes sociais da saúde presentes em gestantes de alto risco? 0 objetivo do presente estudo foi avaliar os determinantes sociais da saúde de gestantes acompanhadas no pré-natal de alto risco.

\section{Métodos}

Estudo de natureza observacional do tipo transversal, desenvolvido em uma maternidade de referência de Fortaleza/CE, Brasil. A unidade é referência para gestação de alto risco e atende mulheres de diversos municípios do estado. A amostra foi selecionada por conveniência e consistiu em 276 gestantes em acompanhamento no ambulatório de pré-natal de alto risco, ou seja, todas as gestantes atendidas no período de agosto a novembro de 2018, com diagnóstico confirmado de alto risco gestacional. Gestantes em surto psicótico ou portadoras de deficiências que a impedissem de responder à pesquisa foram excluídas.

A coleta de dados deu-se mediante aplicação de questionário estruturado, produzido pela própria autora, embasado pelo Modelo de Determinação Social da Saúde de Dahlgren e Whitehead, o qual continha informações acerca das camadas do modelo. A primeira camada relaciona-se aos determinantes individuais - idade, raça, tipo de gestação, trimestre gestacional e problema de saúde prévio. A segunda faz referência ao comportamento e estilo de vida adotado pelo indivíduo - drogas lícitas e/ou ilícitas, atividade física, tempo de sono, atividade sexual e gravidez planejada. A terceira abrange as redes sociais e comunitárias religião e coabitação com o parceiro. A quarta trata dos determinantes intermediários - nível educacional, atividade laboral, renda familiar, situação de moradia, início do pré-natal, internação na gestação e uso de medicamentos. A quinta envolve os determinantes distais, entretanto, optou-se por não abordá-la, por tratar-se de macrodeteminantes de caráter supranacional, tal como a globalização $0^{(4)}$.

Os dados foram compilados no Excel e a análise descritiva por meio do programa estatístico Jamovi®, 
versão 0.9. Verificou-se medianas, intervalo interquartil, frequências absolutas e taxas de prevalência. Optou-se por utilizar a mediana uma vez que $\mathrm{p}<0,05$, conforme o teste de Komolgorov-Smirnov, evidenciando desvio de normalidade. Os resultados foram categorizados e apresentados em tabelas. Posteriormente, foram discutidos conforme o modelo de avaliação dos determinantes sociais da saúde ${ }^{(5)}$.

O estudo foi aprovado pelo Comitê de Ética em Pesquisa da maternidade, conforme recomenda a Resolução no 466/12 do Conselho Nacional de Saúde, que se refere às pesquisas envolvendo seres humanos, sob o Parecer no $2.742 .477 / 2018$.

\section{Resultados}

Os dados coletados foram apresentados em tabelas referentes a cada camada do modelo de determinantes sociais da saúde. A Tabela 1 apresenta a distribuição das gestantes de acordo com os determinantes individuais.

Tabela 1 - Distribuição das gestantes de alto risco de acordo com a primeira camada do modelo de Determinantes Sociais da Saúde. Fortaleza, CE, Brasil, 2018

\begin{tabular}{lc}
$(\mathrm{n}=276)$ & \\
\hline Camada 1 - Determinantes individuais & $\mathbf{n}(\%)$ \\
\hline Idade (anos) & $2(0,7)$ \\
Até 15 & $64(23,2)$ \\
16 a 24 & $139(50,4)$ \\
25 a 34 & $71(25,7)$ \\
$\geq 35$ & \\
Raça & $27(9,8)$ \\
Branca & $17(6,2)$ \\
Preta & $223(80,8)$ \\
Parda & $9(3,2)$ \\
Amarela & \\
Tipo de gestação & $254(92,0)$ \\
Única & $22(8,0)$ \\
Múltipla & \\
Trimestre gestacional & $19(6,9)$ \\
Primeiro & $97(35,1)$ \\
Segundo & $160(58,0)$ \\
Terceiro & \\
Problema de saúde prévio & $155(56,2)$ \\
Sim & $121(43,8)$ \\
Não & \\
\hline
\end{tabular}

A idade das mulheres variou de 15 a 46 anos e a maioria encontrava-se na faixa etária de 25 a 34 anos, 139 (50,4\%). A idade mediana foi de 30 anos, com intervalo interquartílico de 10 . Observou-se predominância da raça parda, 223 (80,0\%), de gestação única, 254 (92\%), de problemas de saúde prévios à gravidez, 155 (56,2\%), e de mulheres no terceiro trimestre gestacional, 160 (58\%). Destaca-se que a idade gestacional variou de nove a 40 semanas, com mediana de 29 e intervalo interquartílico de 13,3 semanas.

A Tabela 2 apresenta a distribuição das gestantes de acordo com o comportamento e estilo de vida e as redes sociais e comunitárias.

Tabela 2 - Distribuição das gestantes de alto risco de acordo com a segunda e a terceira camada do modelo de determinantes sociais da saúde. Fortaleza, CE, Brasil, $2018(n=276)$

\begin{tabular}{|c|c|}
\hline $\begin{array}{l}\text { Camadas } 2 \text { e } 3 \text { - Comportamento e estilo de vida } \\
\text { e redes sociais e comunitárias }\end{array}$ & n (\%) \\
\hline \multicolumn{2}{|l|}{ Drogas lícitas } \\
\hline Sim & $13(4,7)$ \\
\hline Não & $263(95,3)$ \\
\hline \multicolumn{2}{|l|}{ Drogas ilícitas } \\
\hline Sim & $4(1,4)$ \\
\hline Não & $272(98,6)$ \\
\hline \multicolumn{2}{|l|}{ Atividade física } \\
\hline Sim & $29(10,5)$ \\
\hline Não & $247(89,5)$ \\
\hline \multicolumn{2}{|l|}{ Tempo de sono (horas) } \\
\hline$<4$ & $11(4,0)$ \\
\hline 4 a 8 & $165(60,0)$ \\
\hline$>8$ & $100(36,0)$ \\
\hline \multicolumn{2}{|l|}{ Atividade sexual } \\
\hline Sim & $161(58,3)$ \\
\hline Não & $115(41,7)$ \\
\hline \multicolumn{2}{|l|}{ Gravidez planejada } \\
\hline Sim & $108(39,1)$ \\
\hline Não & $167(60,5)$ \\
\hline Dados ausentes & $1(0,4)$ \\
\hline \multicolumn{2}{|l|}{ Religião } \\
\hline Católica & $131(47,5)$ \\
\hline Evangélica & $111(40,2)$ \\
\hline Outras & $4(1,4)$ \\
\hline Não tem & $30(10,9)$ \\
\hline \multicolumn{2}{|l|}{ Coabitação com o parceiro } \\
\hline Sim & $218(79,0)$ \\
\hline Não & $58(21,0)$ \\
\hline
\end{tabular}


No tocante aos comportamentos e ao estilo de vida das gestantes, verificou-se predominância de mulheres não usuárias de drogas lícitas, 263 (95,3\%), e ilícitas, 272 (98,6\%). Considerou-se a ocorrência de tais hábitos pelo menos uma vez durante a gestação. 0 tabagismo foi a prática mais relatada, 10 (3,6\%), seguido do alcoolismo, 7 (2,5\%), e do uso de drogas ilícitas, 4 (1,4\%).

0 sedentarismo foi constante, 247 (89,5\%). Enfatiza-se que $15(5,4 \%)$ gestantes relataram ter cessado as atividades físicas em decorrência da gravidez, seja por medo de perder o bebê ou por restrição médica. 0 hábito de sono foi avaliado a partir do número de horas de sono diário. Observou-se variação de um a 17 horas, com mediana de 8 horas e intervalo interquartílico de 3,8.

Quanto à sexualidade, percebeu-se que a maioria das gestantes apresentava vida sexual ativa, 161 $(58,3 \%)$. No tocante ao planejamento da gravidez, verificou-se predominância do não planejamento, 167 $(60,7 \%)$. Ao que se refere às redes sociais e comunitárias, destaca-se a predominância de gestantes com religião, $246(89,1 \%)$ e que residem com o parceiro, $218(79,0 \%)$.

A Tabela 3, disposta a seguir, destaca a distribuição das gestantes conforme os determinantes intermediários.

A maioria das gestantes possuía de 10 a 12 anos de estudo, 175 (63,4\%), sugerindo escolaridade relativamente boa. Com relação à atividade laboral, observou-se predominância de mulheres do lar, 172 $(62,3 \%)$. Dentre as que exerciam atividade remunerada, menos da metade possuía carteira assinada, 51 $(18,5 \%)$. A renda familiar variou de sem renda a $R \$$ $8.000,00$, com uma mediana de $\mathrm{R} \$ 1.200,00$ e intervalo interquartílico de $\mathrm{R} \$ 1.046,00$. Quanto à situação de moradia, houve predominância de moradia própria, $154(55,8 \%)$.
Tabela 3 - Distribuição das gestantes de alto risco de acordo com a quarta camada do modelo de determinantes sociais da saúde. Fortaleza, CE, Brasil, 2018

\begin{tabular}{|c|c|}
\hline Camada 4 - Determinantes intermediários & n (\%) \\
\hline \multicolumn{2}{|l|}{ Nível educacional (anos) } \\
\hline$\leq 9$ & $59(21,4)$ \\
\hline 10 a 12 & $175(63,4)$ \\
\hline$>12$ & $42(15,2)$ \\
\hline \multicolumn{2}{|l|}{ Atividade laboral } \\
\hline Formal & $51(18,5)$ \\
\hline Informal & $53(19,2)$ \\
\hline Não tem & $172(62,3)$ \\
\hline \multicolumn{2}{|l|}{ Renda familiar (salário mínimo)* } \\
\hline Sem renda & $1(0,3)$ \\
\hline$<1$ & $96(34,8)$ \\
\hline 1 a 3 & $148(53,8)$ \\
\hline$>3$ & $28(10,2)$ \\
\hline Não sabe/não responderam & $3(1,1)$ \\
\hline \multicolumn{2}{|l|}{ Situação de moradia } \\
\hline Própria & $154(55,8)$ \\
\hline Alugada & $94(34,1)$ \\
\hline Cedida & $28(10,1)$ \\
\hline \multicolumn{2}{|l|}{ Início do pré-natal (semanas) } \\
\hline Até 12 & $169(61,2)$ \\
\hline$>12$ & $100(36,2)$ \\
\hline Não informado & $7(2,6)$ \\
\hline \multicolumn{2}{|l|}{ Internação na gestação } \\
\hline Sim & $61(22,1)$ \\
\hline Não & $215(77,9)$ \\
\hline \multicolumn{2}{|l|}{ Uso de medicamentos } \\
\hline Sim & $199(72,1)$ \\
\hline Não & $77(27,9)$ \\
\hline
\end{tabular}

Sob a ótica do acesso aos bens e serviços de saúde, verificou-se, no tocante ao acompanhamento gestacional, que houve predominância de gestantes que iniciaram o pré-natal com até 12 semanas de gravidez. A mediana da idade gestacional no início do pré-natal foi de 11 semanas, com intervalo interquartílico de seis semanas. No que se refere às intercorrências obstétricas, verificou-se que $61(22,1 \%)$ participantes vivenciaram internação hospitalar durante a gestação. Ademais, a maioria da amostra relatou uso de medicamentos, além das vitaminas gestacionais, $199(72,1 \%)$. 


\section{Discussão}

Como limitações, ressalta-se que o presente estudo não realizou avaliação das condições de moradia, uma vez que se tratava de mulheres que residiam na capital, embora seja sabido que a população que reside em zona rural tende a viver em condições mais vulneráveis de saneamento básico. Destaca-se, diante disso, a necessidade de avaliar as iniquidades de saúde relacionadas ao saneamento básico. Adversamente, destaca-se que as análises realizadas poderão contribuir para a elaboração de políticas intersetoriais, que englobem diversos contextos em busca da promoção da saúde, mediante a melhoria das condições de vida dessas mulheres.

Diante das características individuais das gestantes, percebeu-se que a maior parte da amostra encontra-se em uma faixa etária ideal para a reprodução, de 20 a 34 anos. Sabe-se que os extremos de idade materna ( $\leq 15$ ou $\geq 35$ anos) relacionam-se a piores desfechos perinatais ${ }^{(6)}$.

Sob a ótica da obstetrícia, a importância de investigar a raça no cenário dos determinantes sociais da saúde justifica-se pelo fato de haver diferenças entre gestantes de acordo com a etnia. Mulheres africanas possuem maior risco de desenvolvimento de pré-eclâmpsia e vícios pélvicos ${ }^{(7)}$. Logo, a predominância de mulheres pardas foi considerada favorável ao fator de proteção a sua saúde.

Influenciado por fatores genéticos, o tipo de gravidez foi considerado uma característica individual. Sabe-se que gestações múltiplas constituem fator de risco para o desenvolvimento de pré-eclâmp$\operatorname{sia}^{(3)}$. 0 fato da maioria da amostra gestar feto único foi considerado favorável à saúde das gestantes em condição de alto risco.

Quanto às condições clínicas prévias, a maioria das gestantes de alto risco apresentou alguma doença preexistente. Sabe-se que condições clínicas prévias podem relacionar-se a complicações na gestação, assim como a piores desfechos perinatais, podendo interferir na saúde da mulher ${ }^{(8)}$.
No tocante à segunda camada do modelo, no que diz respeito ao uso de drogas lícitas, sabe-se que o uso de drogas e álcool associa-se com incidência de parto prematuro, baixo peso ao nascer e impacto adverso na duração da gravidez ${ }^{(9)}$. Tal achado ratifica a necessidade se conhecer os aspectos não clínicos que interferem na saúde da gestante de alto risco.

No que diz respeito à prática de atividade física, observou-se baixa prevalência. Intervenções baseadas em atividade física associada à dieta resultam em ganho de peso significativamente menor durante a gestação. Além disso, o hábito associa-se de modo relevante à redução da ocorrência de diabetes mellitus gestacional $^{(10)}$. Desse modo, a correta orientação e o estímulo à prática de atividade física podem ser favoráveis à saúde dessas mulheres.

Quanto ao hábito de sono, sabe-se que gestantes que dormem menos de oito horas por dia têm duas vezes mais chance de desenvolver depressão e ansiedade $^{(11)}$, condições capazes de influenciar negativamente sua saúde e bem-estar.

No que tange ao aspecto sexual, embora a maioria das mulheres tenha referido atividade sexual durante a gestação, considerou-se alto o número de gestantes que negaram vida sexual ativa. Ao avaliar gestantes de alto risco na Turquia, evidenciou-se predominância de vida sexual prejudicada a partir do surgimento dos riscos na gestação ${ }^{(12)}$. As principais mudanças apontadas pelas mulheres foram diminuição da frequência de relações sexuais, menor desejo sexual, dor e sofrimento durante o ato, não considerar-se atraente e insatisfação sexual. Percebeu-se que a condição de alto risco gestacional teve um efeito negativo sobre a vida sexual das mulheres ${ }^{(12)}$ e, consequentemente, sobre a sua qualidade de vida. Nesse contexto, percebe-se a importância da educação sexual durante a gestação. Ressalta-se, ainda, a necessidade de técnicas educativas acerca do planejamento da gravidez ${ }^{(13)}$, visto que, no presente estudo, houve predominância de mulheres com gravidez não planejada.

Evidencia-se que a gestação não planejada está diretamente relacionada às condições socioeconômi- 
cas, à vida familiar e conjugal e aos aspectos biopsicossociais. Além disso, percebe-se que, apesar das mulheres terem acesso aos métodos contraceptivos, existe uma lacuna caracterizada pela falta de conhecimento por parte delas ${ }^{(13)}$. Acredita-se que o não planejamento da gestação pode refletir na sua descoberta tardia e no inicio tardio do pré-natal. Logo, interfere no reconhecimento precoce de fatores de risco gestacional $^{(6)}$.

Ademais, sugere-se que quanto maior o número de filhos, maior a chance de preocupações financeiras e desgaste físico, o que implica em uma pior qualidade de vida. 0 elevado número de gestações apresenta associação com a ocorrência de sífilis gestacional, ratificando a necessidade de fortalecer as estratégias de planejamento reprodutivo ${ }^{(14)}$, no intuito de melhorar as condições de saúde dessa população.

Como determinantes da terceira camada, destacam-se as redes sociais e comunitárias, as quais traduzem o nível de coesão social entre as gestantes e a sua família, vizinhos, amigos e grupos, assim como a formação do capital social, abrangendo a sua inter-relação ${ }^{(4)}$.

A gestação retrata uma experiência que pode refletir no comportamento não só da mulher, mas também de sua família e das pessoas com as quais se relaciona, uma vez que consiste em um processo permeado por mudanças físicas, emocionais e sociais. Essas mudanças podem repercutir diretamente na saúde das mulheres. Sob a ótica das gestantes, a participação do companheiro no processo gestatório é essencial, podendo repercutir diretamente no bem-estar materno-fetal ${ }^{(15)}$. Portanto, o fato da maioria das gestantes de alto risco viver com o parceiro é percebido como favorável à sua saúde.

Destacam-se também as relações com grupos religiosos. Aponta-se que o tipo de religião possui relação significativa com a frequência do uso de álcool entre gestantes, de modo que as católicas afirmam maior uso nos finais de semana. Entretanto, a religiosidade pode apresentar-se como fator de proteção na gestação, uma vez que motiva a diminuição do consumo de bebidas alcoólicas e até mesmo a abstinência ${ }^{(16)}$. No tocante aos determinantes intermediários, quanto ao nível educacional, sabe-se que gestantes com maior escolaridade apresentam menor chance de desenvolver sintomas depressivos ${ }^{(17)}$, sendo considerado um fator de proteção à sua saúde. Em contrapartida, o baixo nível de escolaridade é uma barreira para o acesso a informações e é um preditor significativo de mortalidade ${ }^{(18)}$.

Com relação à renda familiar, quando baixa, associa-se a maior incidência de complicações obstétricas, não alcançando informações de qualidade e assistência multiprofissional ${ }^{(18)}$, ratificando a influência da camada econômica sobre o processo saúde-doença. Sobre a situação de moradia, verificamos que as gestantes residiam principalmente em casa própria. Considera-se a situação de moradia um fator determinador para a vulnerabilidade aos agravos de saúde e a outras situações de risco, como o uso de substâncias psicoativas. Mulheres que vivem de aluguel apresentaram 2,8 vezes mais chances de consumir drogas ${ }^{(19)}$. Portanto, considera-se essencial conhecer os determinantes sociais de saúde durante o pré-natal.

Os resultados acerca do início do pré-natal mostraram que a maior parte das gestantes o iniciou antes de 12 semanas, estando em conformidade com a recomendação da política atual de assistência materno-infantil, a Estratégia Rede Cegonha, que visa a ampliação do acesso e a melhoria na qualidade da assistência pré-natal. 0 início precoce do acompanhamento pré-natal favorece o reconhecimento, em tempo oportuno, de condições que oferecem risco ao desenvolvimento da gestação, sendo favorável na prevenção de complicações gestacionais ${ }^{(20)}$.

Com relação ao uso de medicamentos, a maioria da amostra o referiu. Logo, percebe-se a importância de promover o autocuidado entre as mulheres, de modo que compreendam o uso dos remédios, assim como os seus efeitos, e alcancem melhores condições de saúde. 
Embora tenha se observado a conformidade do acesso ao serviço de pré-natal, ainda faz-se necessária a criação de novas políticas intersetoriais de melhorias das condições de vida e de trabalho, que são fundamentais para alcançar a equidade em saúde. Mediante o entendimento de que o alto risco gestacional decorre de diversos fatores, o reconhecimento dos determinantes sociais da saúde, desde as condições individuais até as distais, fornece subsídio para alcançar uma assistência integral à mulher, identificar vulnerabilidades, fomentar novas políticas, com vistas a alcançar melhores resultados materno-fetais, reduzindo as taxas de morbimortalidade dessa população.

\section{Conclusão}

0 presente estudo permitiu o reconhecimento e a avaliação dos determinantes sociais da saúde das gestantes do alto risco. Observou-se que os determinantes individuais - idade, raça e tipo de gestação - foram considerados favoráveis à saúde da maioria das gestantes. Quanto aos determinantes proximais, a maioria das gestantes de alto risco negaram consumo de drogas lícitas e/ou ilícitas, sendo considerado um comportamento favorável. Porém, verificou-se a prevalência de gravidez não planejada como comportamento desfavorável. No que se refere às redes sociais e comunitárias, percebeu-se como favorável o fato da maioria das gestantes viver com parceiro e participar da rede de apoio religioso. No tocante aos determinantes de nível intermediário, verificou-se que o nível educacional, a situação de moradia e o acesso aos serviços de saúde foram determinantes favoráveis à saúde da maioria das mulheres, enquanto a baixa renda consistiu em condição prejudicial no contexto de alto risco.

\section{Colaborações}

Gadelha IP e Aquino PS contribuíram com a concepção e projeto, análise e interpretação dos dados, redação do artigo, revisão crítica relevante do conteúdo intelectual e aprovação final da versão a ser publicada. Diniz FF, Silva DM e Balsells MMD colaboraram na redação do artigo e revisão crítica relevante do conteúdo intelectual. Pinheiro AKB contribuiu com a redação do artigo, revisão crítica relevante do conteúdo intelectual e aprovação final da versão a ser publicada.

\section{Referências}

1. Peixoto S, Mendes ETR. Risco gravídico. In: Peixoto $S$, organizador. Manual de assistência pré-natal. São Paulo: FEBRASGO; 2014. p. 114-19.

2. Word Health Organization. Managing complications in pregnancy and childbirth: a guide for midwives and doctors. Geneva: Word Health Organization; 2017.

3. Ferreira ETM, Moura NS, Gomes MLS, Silva EG, Guerreiro MGS, Oriá MOB. Maternal characteristics and risk factors for preeclampsia in pregnant women. Rev Rene. 2019; 20:e40327. doi: https:// dx.doi.org/10.15253/2175-6783.20192040327

4. Mendes EV. O cuidado das condições crônicas na atenção primária à saúde: o imperativo da consolidação da estratégia da saúde da família. Brasília: Organização Pan-Americana de Saúde; 2012.

5. Dahlgren G, Whitehead M. Policies and Strategies to promote social equity in health. Background document to WHO - Strategy paper for Europe. Stockholm: Institute for Future Studies; 1991.

6. Felczak C, Ravelli APX, Skupien SV, Ricken MH, Bayer LCD, Almeida EA. Profile of cardiac pregnant women: high-risk. Cogitare Enferm. 2018; 23(2):e49605. doi: http://dx.doi.org/10.5380/ ce.v23i2.49605

7. Burton GJ, Redman CW, Roberts JM, Moffett A. Pre-eclampsia: pathophysiology and clinical implications. BMJ. 2019; 366:1-15. doi: https://doi. org/10.1136/bmj.12381

8. Antunes MB, Demitto MO, Gravena AAF, Padovani $C$, Pelloso SM. Hypertensive syndrome and perinatal outcomes in high-risk pregnancies. Rev Min Enferm. 2017; 21:e-1057. doi: http://dx.doi. org/10.5935/1415-2762.20170067 
9. Hamulka J, Zielińska MA, Chądzyńska K. The combined effects of alcohol and tobacco use during pregnancy on birth outcomes. Rocz Panstw Zakl Hig [Internet]. 2018 [cited Oct 13, 2019]; 69(1):45-54. Available in: http://wydawnictwa.pzh.gov.pl/roczniki_pzh/files/pzhissues/ RPZH_2018_Vol_69_No_1_calosc__z_okladka.pdf\#page $=51$

10. International Weight Management in Pregnancy (i-WIP). Collaborative Group. Effect of diet and physical activity based interventions in pregnancy on gestational weight gain and pregnancy Outcomes: meta-analysis of individual participant data from randomized trials. BMJ. 2017; 358:j3119. doi: https://doi.org/10.1136/bmj. j3119

11. Yu Y, Li M, Pu L, Wang S, Wu J, Ruan L, et al. Sleep was associated with depression and anxiety status during pregnancy: a prospective longitudinal study. Arch Women's Mental Health. 2017; 20(5):695-701. doi: https://dx.doi.org/10.1007/ s00737-017-0754-5

12. Tugut N, Golbasi Z, Bulbul T. Quality of sexual life and changes occurring in sexual life of women with righ risk pregnancy. J Sex Marital Ther. 2016; 43(2):132-41. doi: http://dx.doi.org/10.1080/00 92623X.2016.1141816

13. Kornides ML, Kitsantas P, Lindley LL, Wu H. Factors Associated with Young Adult's Pregnancy Liklihood. J Midwifery Womens Health. 2015; 60(2):158-68. doi: https://dx.doi.org/10.1111/ jmwh.12258

14. Macedo VC, Lira PIC, Frias PG, Romaguera LMD, Caires SFF, Ximenes RAA. Risk factors for syphilis in women: case-control study. Rev Saúde Pública. 2017; 51-78. doi: https://doi.org/10.11606/ S1518-8787.2017051007066
15. Martello NV, Wilhelm LA, Cremonese L, Prates LA, Timm MS, Ressel LB. Care practices carried out by the partner in the pregnant woman's. Rev Enferm UFPE on line [Internet]. 2017 [cited Sep 30, 2019]; 11(11):4574-8. Available from: https:// periodicos.ufpe.br/revistas/revistaenfermagem/ article/view/231196/25188

16. Santos MM, Porto PN, Oliveira JF, Pires CGS, Araújo AJS. Associação entre características sociodemográficas e frequência de uso de álcool por gestantes. Rev Baiana Enferm. 2016; 30(2):1-9. doi: http://dx.doi.org/10.18471/rbe.v30i2.14562

17. Lima MOP, Tsunechiro MA, Bonadio IC, Murata M. Depressive symptoms in pregnancy and associated factors: longitudinal study. Acta Paul Enferm. 2017; 30(1):39-46. doi: http://dx.doi. org/10.1590/1982-0194201700007

18. Amjad S, MacDonald I, Chambers T, OsornioVargas A, Chandra S, Voaklander D, et al. Social determinants of health and adverso maternal and birth outcomes $\mathrm{n}$ adolescent pregnancies: $\mathrm{A}$ systematic review and meta-analysis. Paediatr Perinat Epidemiol. 2019; 33(1):88-99. doi: http:// dx.doi.org/10.1111/ppe.12529

19. Porto PN, Borges SAC, Araújo AJS, Oliveira JS, Almeida MS, Pereira MN. Factors associated with the use of alcohol and drugs by pregnant women. Rev Rene. 2018; 19:e3116. doi: https://doi. org/10.15253/2175-6783.2018193116

20. Balsells MMD, Oliveira TMF, Bernardo EBR, Aquino PS, Damasceno AKC, Castro RCMB, et al. Evaluation of prenatal care process for habitual-risk pregnant women. Acta Paul Enferm. 2018; 31(3):247-54. doi: http://dx.doi. org/10.1590/1982-0194201800036

\section{(cc) BY}

Este é um artigo de acesso aberto distribuído sob os termos da Licença Creative Commons

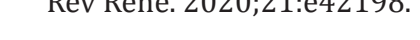

